\title{
吡嗪-噻唑联芳类化合物的合成及抗氧化性能研究
}

\author{
张晓平 $*, a$ 金桂勇 $a$ 陈芝飞 $b$ 王清福 $b$ 赵森森 $b$ 武志勇 $a$ \\ 万帅 $b$ 席高䂞*,b赵旭*,b \\ $(a$ 河南农业大学烟草学院 郑州 450002) \\ ( $b$ 河南中烟工业有限责任公司技术中心 郑州 450000)
}

\begin{abstract}
摘要 为了从吡嗪和噻唑类化合物中寻找新的抗氧化剂, 通过活性基团拼接方法, 设计合成了一系列吡嗪-噻唑联芳 类化合物. 借助 ${ }^{1} \mathrm{H} N M R 、{ }^{13} \mathrm{C} N M R 、 I R$ 和 HPLC-MS 等手段对目标化合物的结构进行了表征，并通过抑制自由基引发 DNA 氧化反应及淬灭自由基反应体系对化合物的抗氧化活性和还原能力进行了测试. 结果表明, 在 5 个测试体系中, 8 个目标化合物均能够有效抑制自由基引发 DNA 氧化反应并捕获自由基, 具有较强自由基的清除能力和还原能力, 是一 类潜在的抗氧化剂. 其中, 在抑制 2,2'偶氮二异丁基椫二盐酸盐(AAPH)引发 DNA 氧化反应体系中, 8 个化合物的有效 计量因子 $(n)$ 可达 1.48 2.12; 在抑制 $\mathrm{HO} \bullet$ 和还原型谷胱甘肽自由基(GS•)引发 DNA 氧化反应体系中, 8 个化合物相对空 白硫代巴比妥酸(TBARS)吸光度百分数分别为 $54.3 \% \sim 76.1 \%$ 和 $55.4 \% \sim 68.3 \% ; 8$ 个化合物均能够捕获 $2,2^{\prime}$-偶氮-双-(3乙基苯并噻唑啉-6-磺酸)二铵盐自由基(ABTS•)和二苯苦味酰胇自由基(DPPH•). 另外, 对比发现吡嗪-噻唑联芳类化合 物表现出优于吡嗪-噁唑联芳类化合物的抗氧化性能.
\end{abstract}

关键词＼cjkstart吡嗪-噻唑; 氧化偶联; 抗氧化; DNA; 自由基

\section{Synthesis and Antioxidant Properties of Pyrazine-Thiazole Bi-heteroaryl Compounds}

\author{
Zhang, Xiaoping ${ }^{*, a} \quad$ Jin, Guiyong ${ }^{a} \quad$ Chen, Zhifei $^{b} \quad$ Wang, Qingfu ${ }^{b} \quad$ Zhao, Sensen $^{b}$ \\ Wu, Zhiyong ${ }^{a} \quad$ Wan, Shuai $^{b} \quad \mathrm{Xi}$, Gaolei*,b Zhao, $\mathrm{Xu}^{*, b}$ \\ $\left({ }^{a}\right.$ College of Tobacco Science, Henan Agricultural University, Zhengzhou 450002) \\ $\left({ }^{b}\right.$ Technology Center, China Tobacco Henan Industrial Co., Ltd., Zhengzhou 450000)
}

\begin{abstract}
In order to find novel pyrazine- and thiazole-based derivatives with potent antioxidant properties, eight pyrazine-thiazole bi-heteroaryl compounds were designed and prepared via active group splicing method between pyrazine- $N$ oxides and thiazoles. They were structurally characterized by ${ }^{1} \mathrm{H}$ NMR, ${ }^{13} \mathrm{C}$ NMR, IR, and HPLC-MS. Antioxidant abilities of the obtained compounds were evaluated by inhibiting radicals induced oxidation of DNA and quenching radicals. The results showed that eight compounds can effectively inhibit radicals induced oxidation of DNA and quench radicals, which revealed that the compounds have strong radical scavenging properties and reduction ability, and can be potential antioxidants. The effective measurement factor $(n)$ values of these compounds ranged from 1.48 to 2.12 in 2,2-azobis(2-amidinopropanehydrochloride) (AAPH) induced oxidation of DNA. The absorbance percentages of eight compounds to the blank thiobarbituric acid reactive substance (TBARS percentage) were $54.3 \% \sim 76.1 \%$ and $55.4 \% \sim 68.3 \%$ in inhibiting $\mathrm{HO} \bullet$ and glutathione radical (GS•) induced oxidation of DNA, respectively. Eight compounds can scavenge 2,2-azinobis(3-ethylbenzothiazoline-6-sulfonate) cationic radical (ABTS•) and 2,2-diphenyl-1-picrylhydrazyl radical (DPPH•). In addition, it was found that the antioxidant activity of pyrazine-thiazole bi-heteroaryl compounds was significantly higher than that of pyrazine-oxazole bi-heteroaryl compound.
\end{abstract}

Keywords pyrazine-thiazole; oxidative cross-dehydrogenative coupling; antioxidant; DNA; free radical

*Corresponding authors. E-mail: xigaoleijida@126.com; zhaoxuzzu@163.com; xiaoping078@163.com

Received November 9, 2020; revised January 11, 2021; published online February 3, 2021.

Project supported by the Young Talents Program of Henan Agricultural University (No. 30500602).

河南农业大学青年英才(No. 30500602)资助项目. 


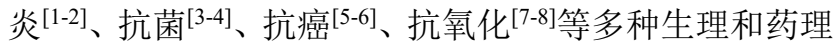
活性. 因此，吡嗪和噻唑类衍生物的合成及生物活性探 索仍为当前研究热点 ${ }^{[9-10]}$. 迄今为止, 国内外关于兼具 吡嗪和噻唑双杂环骨架结构的化合物抗氧化性能研究 报道较少, 研究主要集中于吡嗪-噻唑联芳类化合物的 合成及催化剂的耖选. 当前选取的催化剂以金属钯 ${ }^{[11-12]}$ 和金属有机碱 $[13]$ 为主, 存在反应条件苛刻、产率低、成 本高等缺点. 因此, 有必要开展吡嗪一噻唑联芳类化合 物的高效合成及抗氧化性能研究.

本文在前期研究 ${ }^{[14]}$ 基础上，对合成催化剂进行篎 选, 最终以吡嗪- $N$-氧化物和噻唑化合物为原料, $\mathrm{FeCl}_{3}$ 为催化剂, 经氧化偶联反应高效合成多种双杂环天然骨 架结构的吡嗪-噻唑联芳化合物. 采用多种自由基氧化 DNA 的反应体系检测其抗氧化活性, 并通过淬灭自由 基实验考察其还原自由基能力, 进而探究吡嗪-噻唑联 芳化合物的抗氧化构效关系, 旨在为抗氧化剂的开发及 应用提供理论参考.

\section{1 结果与讨论}

\section{1 化合物的合成}

以化合物 $3 \mathbf{a}$ 的合成为例, 探讨了不同的反应条件 对 3a 产率的影响. 由表 1 可以看出, 选用 1,2-二氯乙烷 作溶剂, 以 $\mathrm{FeCl}_{3}$ 为催化剂, 加热回流反应 $8 \mathrm{~h}$, 是合成 目标化合物的较佳方法，化合物 $3 \mathrm{a}$ 的产率为 $64 \%$. Khake 等 ${ }^{[11]}$ 以 1,4 -二氧六环为溶剂, $\mathrm{CsF}$ 和 $4 \mathrm{~A}$ 分子篎为 添加剂, 利用金属钯催化 2-氯吡嗪和 2-(三正丁基锡基) 噻唑发生交叉偶联反应合成吡嗪一噻唑联芳类化合物 (Scheme 1a); Pandey 等 ${ }^{[12]}$ 以 DMF 为溶剂, $\mathrm{CuI}$ 和 $\mathrm{K}_{3} \mathrm{PO}_{4}$ 为添加剂, 采用金属钯催化 2-碘吡嗪与苯并噻唑发生交 叉偶联反应合成吡嗪一噻唑联芳类化合物(Scheme 1b); $\mathrm{Wu}$ 等 ${ }^{[13]}$ 曾选取吡嗪- $N$-氧化物和噻唑化合物作原料, 四 丁基溴化铵 (TBAB) 为添加剂, 在二甲苯溶剂中以 ${ }^{t} \mathrm{BuOLi}$ 为催化剂合成吡嗪-噻唑联芳类化合物(Scheme $1 \mathrm{c})$. 但这些方法反应条件苛刻、成本高, 所得化合物 $\mathbf{3 a}$ 的产率最高为 $46 \%$, 明显低于本文的产率. 因此, 本文 方法具有操作简单, 成本低, 产率高等优点. 采用该方 法合成了其它目标化合物, 并通过 ${ }^{1} \mathrm{H} N \mathrm{NM} 、{ }^{13} \mathrm{C} \mathrm{NMR}$ 、 IR 和 UHPLC-ESI-MS 等手段对化合物结构进行了表征.

\section{2 化合物的抗氧化活性}

\subsection{1 化合物抑制 AAPH 引发 DNA 氧化反应分析}

不同化合物 TBARS 吸光度随反应时间的变化如图 1 所示. 当加入的反应原料浓度从 $100 \mu \mathrm{mol} / \mathrm{L}$ 至 400 $\mu \mathrm{mol} / \mathrm{L}(4$ 个吡嗪化合物 $\mathbf{1 a} \sim \mathbf{1 d}$ 和 5 个噻唑化合物 $\mathbf{2 a} \sim$ 2d)变化时, TBARS 吸光度曲线与空白实验相比有所降 (a)

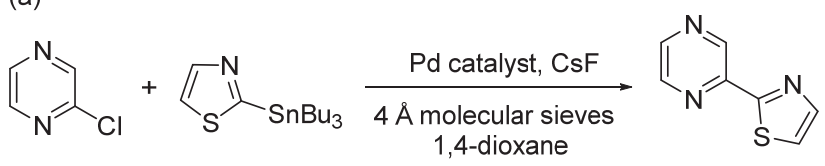

(b)

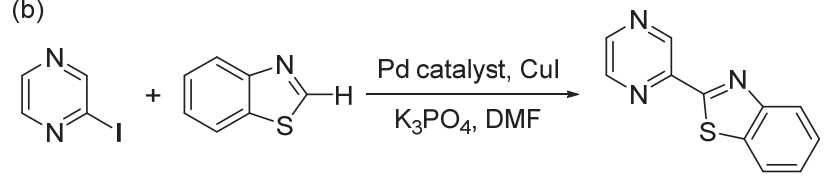

(c)

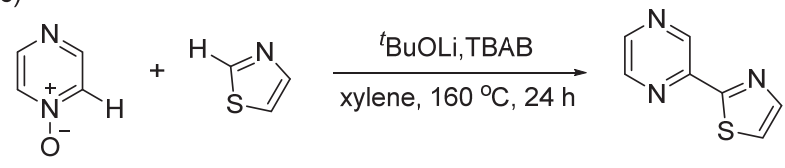

图式 1 吡嗪-噻唑联芳类化合物的合成方法

Scheme 1 Synthetic methods of pyrazine-thiazole bi-heteroaryl compounds

表 1 不同反应条件对目标化合物 3a 合成产率的影响

Table 1 Effects of reaction conditions on the synthesis of the target compound 3a

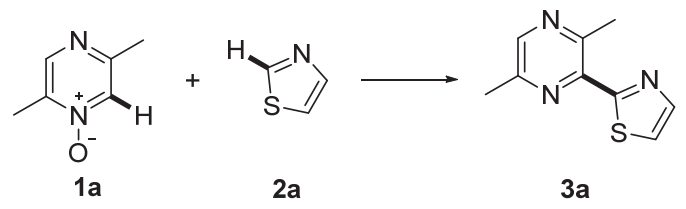

\begin{tabular}{clllllc}
\hline Entry & \multicolumn{1}{c}{ Catalyst } & \multicolumn{1}{c}{ Solvent } & Time/h & T/ ${ }^{\circ} \mathrm{C}$ & Yield/\% \\
\hline 1 & $\mathrm{FeCl}_{3}$ & Toluene & 6 & Reflux & 31 \\
2 & $\mathrm{FeCl}_{3}$ & $\mathrm{CH}_{3} \mathrm{CN}$ & 6 & Reflux & 37 \\
3 & $\mathrm{FeCl}_{3}$ & $\mathrm{CHCl}_{3}$ & 6 & Reflux & 47 \\
4 & $\mathrm{FeCl}_{3}$ & $\mathrm{ClCH}_{2} \mathrm{CH}_{2} \mathrm{Cl}$ & 6 & Reflux & 61 \\
5 & $\mathrm{FeCl}_{3}$ & $\mathrm{ClCH}_{2} \mathrm{CH}_{2} \mathrm{Cl}$ & 8 & Reflux & 64 \\
6 & $\mathrm{FeCl}_{3}$ & $\mathrm{ClCH}_{2} \mathrm{CH}_{2} \mathrm{Cl}$ & 10 & Reflux & 62 \\
7 & $\mathrm{FeCl}_{3}$ & $\mathrm{ClCH}_{2} \mathrm{CH}_{2} \mathrm{Cl}$ & 8 & 60 & 39 \\
8 & $\mathrm{FeCl}_{3}$ & $\mathrm{ClCH}_{2} \mathrm{CH}_{2} \mathrm{Cl}$ & 8 & 25 & Trace \\
9 & ${ }^{t} \mathrm{BuOLi}^{2} \mathrm{TBAB}^{[13]}$ & $\mathrm{Xylene}$ & 24 & Reflux & 46 \\
10 & $\mathrm{InCl}_{3}$ & $\mathrm{ClCH}_{2} \mathrm{CH}_{2} \mathrm{Cl}$ & 8 & Reflux & 31 \\
11 & $\mathrm{BiCl}_{3}$ & $\mathrm{ClCH}_{2} \mathrm{CH}_{2} \mathrm{Cl}$ & 8 & Reflux & 34 \\
12 & $\mathrm{LaCl}_{3}$ & $\mathrm{ClCH}_{2} \mathrm{CH}_{2} \mathrm{Cl}$ & 8 & Reflux & 25 \\
13 & $\mathrm{CeCl}_{3}$ & $\mathrm{ClCH}_{2} \mathrm{CH}_{2} \mathrm{Cl}$ & 8 & Reflux & 37 \\
14 & $\mathrm{YbCl}_{3}$ & $\mathrm{ClCH}_{2} \mathrm{CH}_{2} \mathrm{Cl}$ & 8 & Reflux & 33 \\
15 & $\mathrm{ScCl}_{3}$ & $\mathrm{ClCH}_{2} \mathrm{CH}_{2} \mathrm{Cl}$ & 8 & Reflux & 26 \\
16 & $\mathrm{CuCl}_{2}$ & $\mathrm{ClCH}_{2} \mathrm{CH}_{2} \mathrm{Cl}$ & 8 & Reflux & 21 \\
17 & $\mathrm{AgCl}$ & $\mathrm{ClCH}_{2} \mathrm{CH}_{2} \mathrm{Cl}$ & 8 & Reflux & 12 \\
\hline
\end{tabular}

低，但走向与空白实验基本相同，说明它们不具有抑制 AAPH 氧化 DNA 的活性. 当 8 个目标化合物加入后, TBARS 曲线在反应开始阶段升速变慢, 表明这些化合 

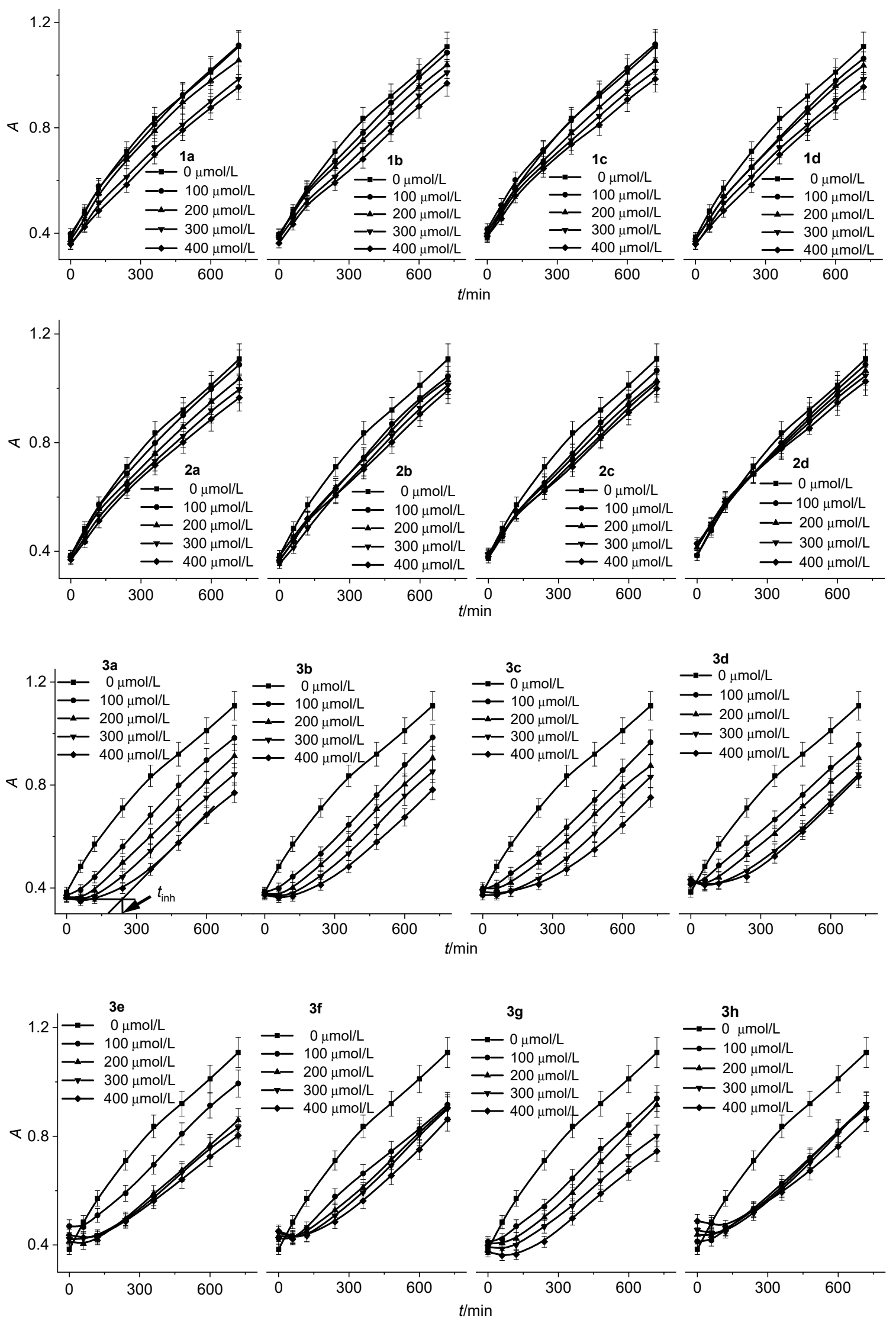

图 1 不同浓度化合物存在下 TBARS 吸光度随时间的变化曲线

Figure 1 Variation of the absorbance of TBARS in various concentrations compounds 
物能够抑制 DNA 的氧化反应，并产生一段抑制期 $\left(t_{\mathrm{inh}}\right)$, 说明这些化合物发挥抗氧化作用. 化合物 $3 \mathbf{a} \sim \mathbf{3 h}$ 抑制 期 $\left(t_{\mathrm{inh}}\right)$ 与浓度的关系曲线如图 2 所示.

化合物 3a 3h 抑制期 $\left(t_{\mathrm{inh}}\right)$ 与浓度的方程式, 以及 有效剂量因子 $(n)$ 如表 2 所示. 为了更直观地表达各个化 合物的有效计量因子 $(n)$ 与结构之间的关系, 将化合物 结构和其 $n$ 值集成于图 3 中. 化合物 $\mathbf{3 a}$ 的 $n$ 值为 1.78 , 表明每个分子 $3 \mathrm{a}$ 能够捕获 1.78 个自由基. 从分子结构 看, 化合物 $3 \mathbf{a}$ 分子骨架中未含传统的抗氧化官能团(羟 基、氨基、巯基和二茂铁基等), 但却展现了一定的抗氧 化能力, 说明羟基、氨基和二茂铁基等不是吡嗪-噻唑联 芳类化合物抑制 DNA 氧化必须的官能团, 其结构骨架 本身能够抑制 AAPH 氧化 DNA. 化合物 3a 的 $n$ 值大于 文献[14]报道的吡嗪-啞唑联芳类化合物 IIIa, 说明噻唑 环比噁唑环更有利于联芳类化合物抑制 AAPH 氧化 DNA. 化合物 $\mathbf{3 b}$ 和 $\mathbf{3 c}$ 的 $n$ 值大于化合物 $\mathbf{3 a}$, 且化合物 3c 的 $n$ 值最大(1.92), 说明 $\mathrm{CH}_{3}$ 能够提高化合物抑制 AAPH 氧化 DNA 的能力, 而且随着 $\mathrm{CH}_{3}$ 数量的增加化 合物的抗氧化性能不断提高 ${ }^{[14]}$. 化合物 $3 \mathbf{d}$ 的 $n$ 值为 2.12 , 是 8 个化合物中最大的, 说明增加共轭结构能显 著提高化合物抑制 AAPH 氧化 DNA 的能力 ${ }^{[15]}$. 化合物 $3 \mathbf{e}$ 的 $n$ 值(1.81)略高于 $\mathbf{3 a}$, 说明 $\mathrm{CH}_{3}$ 位置改变能够影响 化合物抑制 AAPH 氧化 DNA. 化合物 $3 \mathbf{f}$ 的 $n$ 值(1.85)
大于 $3 \mathbf{e}$ 的 1.81 , 说明增大取代基的给电子能力能够提 高化合物抑制 AAPH 氧化 DNA 的能力. 与化合物 $\mathbf{3 a}$ 相 比, 化合物 $\mathbf{3 g}$ 的 $n$ 值增加至 2.05, 进一步化合物 $\mathbf{3 h}$ 的 $n$ 值增加至 2.12, 再次说明增加共轭程度或增加 $\mathrm{CH}_{3}$ 能提 高化合物抑制 AAPH 氧化 DNA 的能力.

\subsection{2 抑制 $\mathrm{HO} ・$ 和 $\mathrm{GS}$ •引发 DNA 氧化反应体系分析}

化合物抑制 $\mathrm{HO}$ ・和 $\mathrm{GS}$ •引发 DNA 氧化反应的 TBARS 吸光度百分数, 如图 3 所示. 由图 3 可知, 在抑 制 HO•引发 DNA 氧化反应体系中, 8 个原料(4 个吡嗪化 合物 $\mathbf{1 a} \sim \mathbf{1 d}$ 和 4 个噻唑化合物 $\mathbf{2 a \sim 2 d}$ ) 和 8 个吡嗪-噻 唑联芳类化合物 $(\mathbf{3 a} \sim \mathbf{3 h})$ 相对空白 $\mathrm{TBARS}$, 吸光度百 分数均低于 $100 \%$ ，表明 16 个化合物均具备抑制 $\mathrm{HO} \cdot$ 氧 化 DNA 的活性. 8 个原料的 TBARS 百分数均大于 $87 \%$, 而 8 个目标化合物 $\mathbf{3 a} \sim \mathbf{3 h}$ 的 TBARS 百分数均小于 $73.4 \%$ ，说明目标化合物抑制 HO•氧化 DNA 的活性远高 于原料. 其中, 化合物 $\mathbf{3 a}$ 的 TBARS 百分数为 $68.7 \%$, 文 献[14]报道吡嗪-噁唑联芳类化合物(IIIa)的 TBARS 百 分数为 $76.1 \%$, 说明噻唑环作为取代基更有利于化合物 抑制 HO•氧化 DNA，与抑制 AAPH 氧化 DNA 体系分析 结果一致. 化合物 $\mathbf{3 b}$ 和 $\mathbf{3 c}$ 的 TBARS 百分数大于化合 物 3a，说明引入甲基使化合物抑制 $\mathrm{HO}$-氧化 DNA 的能 力降低, 而且随着 $\mathrm{CH}_{3}$ 数量的增加, 整个分子的抗氧化 性能不断降低. 化合物 $\mathbf{3 d}$ 和 $\mathbf{3 g}$ 的 TBARS 百分数明显

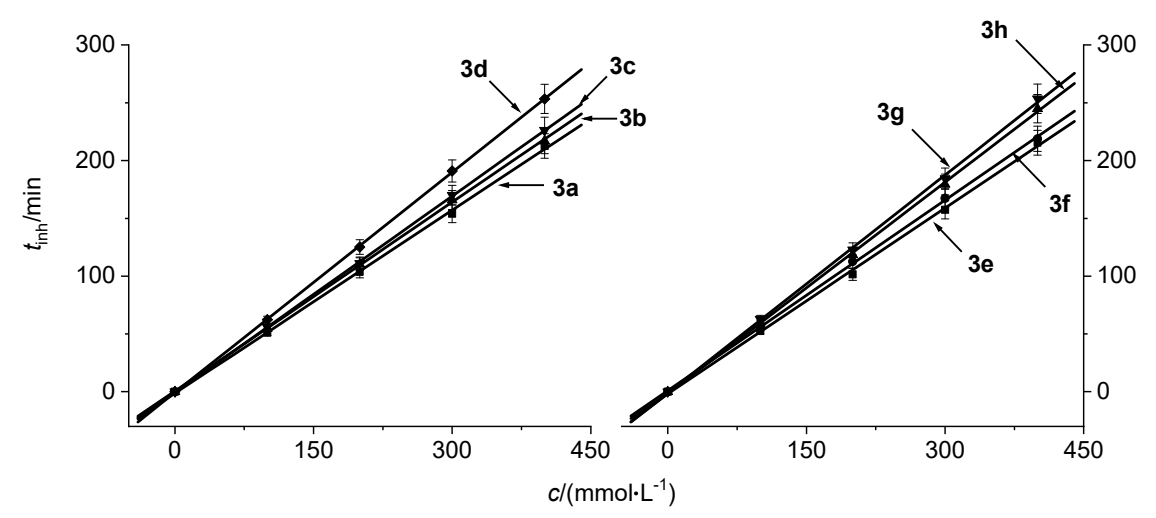

图 2 抑制期 $\left(t_{\mathrm{inh}}\right)$ 与化合物浓度之间的关系

Figure 2 Relationships between inhibition period $\left(t_{\mathrm{inh}}\right)$ and concentrations of compounds

表 2 化合物抑制期 $\left(t_{\mathrm{inh}}\right)$ 与浓度 $(c)$ 关系方程式以及 $n$ 值

Table 2 Equations of inhibition period $\left(t_{\text {inh }}\right)$ and compound concentration $(c)$, and $n$ of compounds

\begin{tabular}{ccc}
\hline Compd. & $t_{\text {inh }}(\min )=\left(n / R_{\mathrm{i}}\right) c_{\text {compound }}+$ constant & $n$ \\
\hline 3a & $t_{\text {inh }}=0.53( \pm 0.03) c(\mathbf{3 a})-1.34( \pm 0.07)$ & $1.78( \pm 0.08)$ \\
3b & $t_{\text {inh }}=0.54( \pm 0.03) c(\mathbf{3 b})+0.59( \pm 0.03)$ & $1.81( \pm 0.09)$ \\
3c & $t_{\text {inh }}=0.57( \pm 0.03) c(\mathbf{3 c})-0.73( \pm 0.04)$ & $1.92( \pm 0.10)$ \\
3d & $t_{\text {inh }}=0.63( \pm 0.03) c(\mathbf{3 d})+0.68( \pm 0.03)$ & $2.12( \pm 0.11)$ \\
3e & $t_{\text {inh }}=0.54( \pm 0.03) c(\mathbf{3 e})-1.72( \pm 0.09)$ & $1.81( \pm 0.09)$ \\
3f & $t_{\text {inh }}=0.55( \pm 0.03) c(\mathbf{3 f})+0.96( \pm 0.05)$ & $2.05( \pm 0.10)$ \\
3g & $t_{\text {inh }}=0.61( \pm 0.03) c(\mathbf{3 g})-1.66( \pm 0.08)$ & $2.12( \pm 0.11)$ \\
3h & $t_{\text {inh }}=0.63( \pm 0.03) c(\mathbf{3 h})-0.99( \pm 0.05)$ & \\
\hline
\end{tabular}



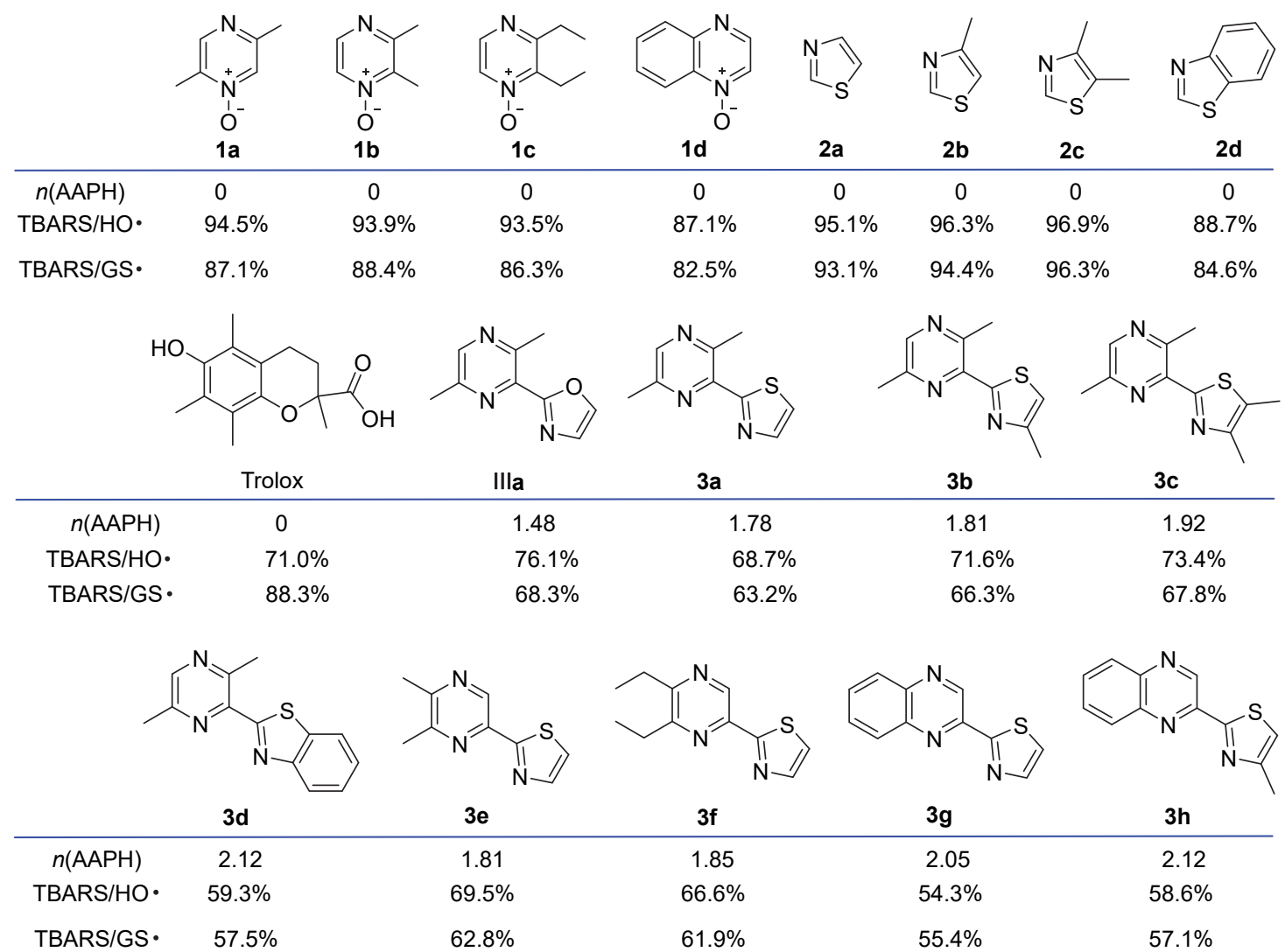

图 3 化合物抑制 AAPH、HO・和 GS・引发 DNA 氧化反应活性

Figure 3 Properties of compounds in inhibiting AAPH, HO•and GS• induced oxidation of DNA.

小于化合物 $\mathbf{3 a}$, 说明无论在吡嗪环还是噻唑环上增大 共轭体系, 均有利于化合物抑制 HO-氧化 DNA. 化合物 $3 \mathrm{f}$ 的 TBARS 百分数分别为 $66.6 \%$, 小于 $3 \mathrm{e}$ 的 $69.5 \%$, 说 明增大取代基的给电子能力有利于化合物抑制 $\mathrm{HO}$-氧 化 DNA. 化合物 $\mathbf{3 h}$ 的 TBARS 百分数为 $58.6 \%$, 大于 $\mathbf{3 g}$ 的 54.3\%, 再次说明引入甲基不利于化合物抑制 $\mathrm{HO}$-氧 化 DNA. 由图 3 可知, 在抑制 GS • 引发 DNA 氧化反应 中, 8 个联芳类化合物所得实验结果与 $\mathrm{HO}$. 引发 DNA 的 氧化反应体系相同, 进一步证明增大化合物的共轭体系 能够提供更多与 $\mathrm{HO}$ •和 GS・发生自由基加成的位点, 从 而展现更好的抑制自由基氧化 DNA 性能 ${ }^{[16]}$, 而引入甲 基使共轭体系官能团增多, 从空间效应上不利于 $\mathrm{HO}$ • 和 $\mathrm{GS} \cdot$ 与苯环的自由基加成反应 ${ }^{[16]}$.

\section{3 化合物的还原自由基性能}

$\mathrm{ABTS} ・$ 和 $\mathrm{DPPH} \cdot$ 浓度随时间的变化曲线如图 4 所 示. 在含有化合物 $\mathbf{1 a} \sim \mathbf{1 d}$ 和 $\mathbf{2 a} \sim \mathbf{2 d}$ 体系中, ABTS•和 $\mathrm{DPPH} \cdot$ 浓度随时间的增长均未降低, 与空白体系相同, 说明 8 个原料不具有淬灭 ABTS • 和 $\mathrm{DPPH} \bullet$ 的能力. 当加 入目标化合物 $\mathbf{3 a} \sim \mathbf{3 h}$ 后, ABTS•和 DPPH•的浓度随反应 时间的增长均逐渐减小, 说明 8 个吡嗪一噻唑联芳类化 合物能够提供电子与 $\mathrm{ABTS}$ ・和 $\mathrm{DPPH} \cdot$ 孤对电子配对,
进而清除 $\mathrm{ABTS}$ ・和 $\mathrm{DPPH} \bullet$. 为了更直观比较化合物清 除自由基性能，由 ABTS・和 DPPH・浓度随时间变化曲 线求得目标化合物 $\mathbf{3 a} \sim \mathbf{3 h}$ 与 $\mathrm{ABTS} \cdot$ 和 $\mathrm{DPPH}$ •反应 1800 $\mathrm{s}$ 的自由基清除率, 如图 5 所示.

化合物 3a 对 ABTS•清除率为 8.66\%, 高于化合物 IIIa 的 $6.37 \%$, 说明吡嗪-噻唑联芳类化合物清除 ABTS能力优于吡嗪-噁唑联芳类化合物. 化合物 $\mathbf{3 b}$ 和 $\mathbf{3 c}$ 对 ABTS-清除率分别为 $9.89 \%$ 和 $11.24 \%$, 高于化合物 3a, 且化合物 $3 \mathbf{c}$ 数值最大, 说明引入 $\mathrm{CH}_{3}$ 有利于化合物捕 获 ABTS•, 且随着 $\mathrm{CH}_{3}$ 数量的增加, 化合物捕获 ABTS• 的能力不断增强. 化合物 $3 \mathrm{~g}$ 和 $3 \mathrm{~h}$ 对比结果进一步验证 了该结论. 化合物 $\mathbf{3 d}$ 和 $\mathbf{3 g}$ 对 $\mathrm{ABTS} \cdot$ 清除率分别为 $12.28 \%$ 和 $12.07 \%$ ，明显高于化合物 $\mathbf{3 a}$, 说明增大化合 物的共轭体系能提高吡嗪一噻唑联芳类化合物淬灭 $\mathrm{ABTS} \bullet$ 的能力, 与抑制自由基引发 DNA 氧化反应一致. 化合物 3e 对 ABTS•清除率为 $9.47 \%$, 略高于化合物 3a, 说明 $\mathrm{CH}_{3}$ 位置的改变也能影响吡嗪-噻唑联芳类化合物 捕获 ABTS • 的能力. 化合物 $3 \mathbf{f}$ 对 ABTS • 清除率为 $10.51 \%$ ，高于化合物 $3 \mathrm{e}$, 说明增大取代基的给电子能力 能够提高化合物淬灭 ABTS • 的能力. 由图 5 可知, 吡嗪噻唑联芳类化合物清除 $\mathrm{DPPH} \bullet$ 结果与捕获 $\mathrm{ABTS}$ •反应 

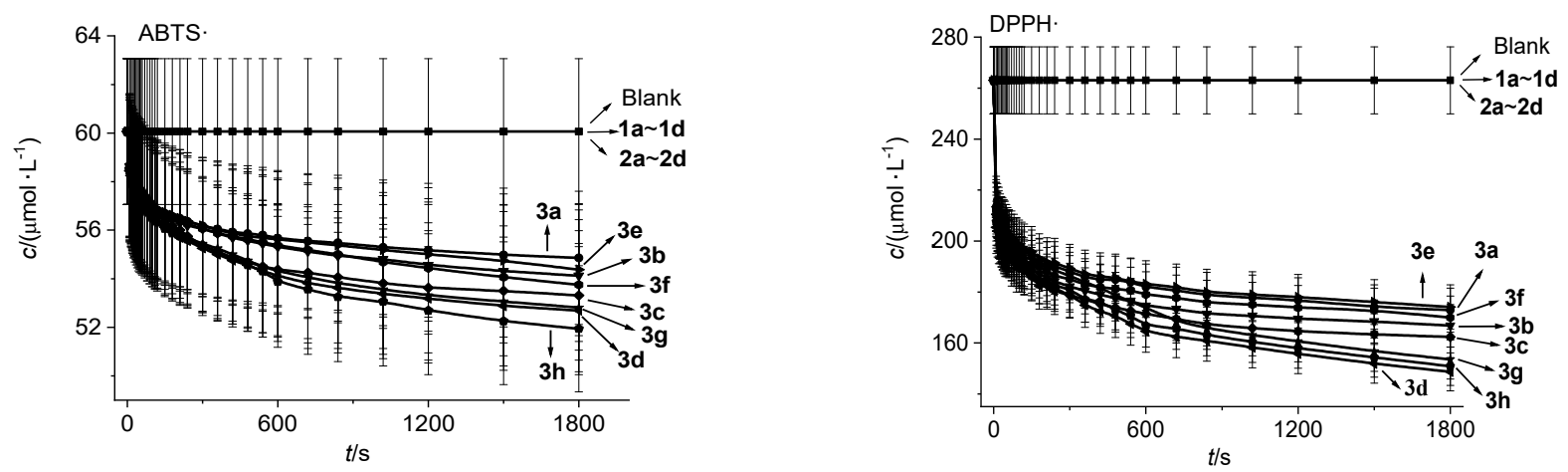

图 4 在不同化合物存在下 ABTS•和 $\mathrm{DPPH} \bullet$ 浓度随时间的衰减曲线

Figure 4 Decay of concentrations of ABTS• and DPPH• in the presence of compounds

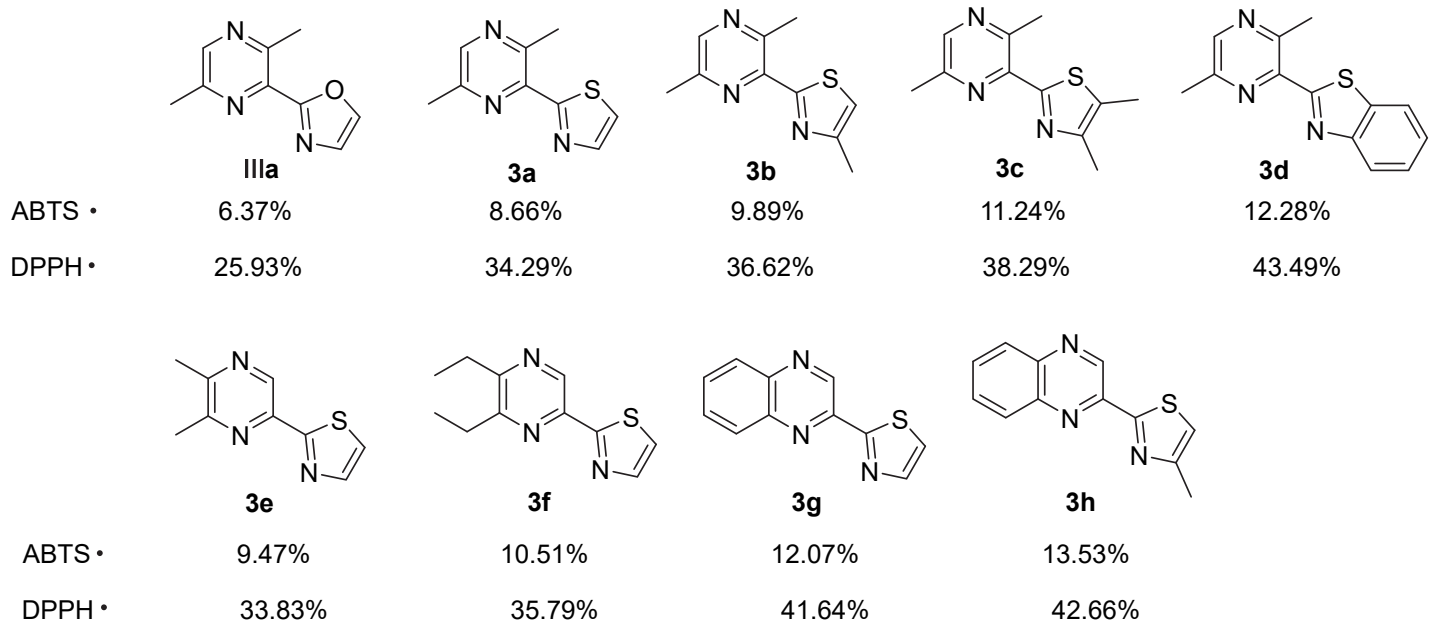

图 5 化合物清除 $\mathrm{ABTS}$ ・和 $\mathrm{DPPH} ・$ 性能

Figure 5 Properties of compounds in scavenging ABTS• and DPPH• radicals

体系相同，进一步证明引入甲基、增大化合物共轭体系 或增强取代基的给电子能力均能提高化合物清除自由 基的能力.

\section{2 结论}

本文采用活性基团拼接方法, 合成了 8 个吡嗪一噻 唑联芳类化合物. 初步的抗氧化性能测试结果表明, 8 个联芳类化合物在 5 个体系中均展现了一定的抗氧化活 性，是潜在的抗氧化剂，具有广阔的应用前景. 其中， 在 5 个测试体系中, 化合物 $3 \mathrm{~d} 、 3 \mathrm{~g}$ 和 $3 \mathrm{~h}$ 表现出明显优 于其它 5 个化合物的抗氧化性能; 在抑制 AAPH 引发 DNA 氧化反应及淬灭 $\mathrm{ABTS} \cdot$ 和 $\mathrm{DPPH} \cdot$ 体系中，化合物 3b、3c 和 $3 \mathbf{f}$ 的性能高于化合物 3a 的性能; 在抑制 HO和 GS• 引发 DNA 氧化反应体系中, 化合物 $3 \mathbf{a}$ 则表现出 优于化合物 $3 \mathrm{~b}$ 和 $3 \mathrm{c}$ 的性能. 这些实验数据为今后继续 从事吡嗪-噻唑联芳类化合物的合成及抗氧化性能研究 提供了理论参考.

\section{3 实验部分}

\section{1 仪器与试剂}

分析天平 $(0.0001 \mathrm{~g})$, 德国 Sartorius 公司; HH-6 恒温 水浴锅, 江苏金坛市宏华仪器厂; DF-101S 集热式恒温 加热磁力摚拌器, 河南省巩义市予华仪器有限责任公 司; SHZ-D(III)循环水式真空百, 河南省巩义市予华仪 器有限责任公司; 旋转蒸发仪, 德国 IKA 集团; JH40 全 自动熔点仪, 上海佳航仪器仪表有限公司; Bruker Avance AMX-400 核磁共振谱仪, 美国 Bruker 公司; 傅 立叶变换红外光谱仪, 美国 Perkin-Elme 公司; 超高效 液相色谱一电喷雾离子源一质谱联用仪, 美国 Agilent technologies 公司; UV1101 紫外可见分光光度计, 天美 (中国)科学仪器有限公司.

2,5-二甲基吡嗪- $N$-氧化物(1a)、2,3-二甲基吡嗪- $N$ 氧化物(1b)、2,3-二乙基吡嗪- $\mathrm{N}$-氧化物(1c)、喹喔啉- $N$ 氧化物(1d)、噻唑(2a)、4-甲基噻唑(2b)、4,5-二甲基噻 唑(2c)、苯并噻唑(2d), 分析纯, 北京化工厂; 无水三氯 
化铁、2-硫代巴比妥酸(TBA), 分析纯, 国药集团化学试 剂有限公司; 脱氧核糖核酸钠盐(DNA)、2,2'-偶氮二异 丁基脒二盐酸盐(AAPH), 优级纯, ACROS ORGANICS 公司; 还原型谷胱甘肽(GSH)、四氯氢醌(TCHQ), 分析 纯，上海源叶生物科技有限公司; 三氯乙酸(TCA)、乙二 胺四乙酸二钠(EDTA)、正丁醇、二甲基亚砜(DMSO), 分 析纯, 上海达瑞精细化学品有限公司; 2,2'-偶氮-双-(3乙基苯并噻唑啉-6-磺酸)二铵盐自由基(ABTS•)、二苯苦 味酰肼自由基(DPPH•), 优级纯, 美国 Sigma-Aldrich 公 司

\section{2 吡嗪 - 噻唑联芳化合物 $3 a \sim 3 h$ 的合成}

在 $100 \mathrm{~mL}$ 圆底烧瓶中, 加入含不同取代基的吡嗪$N$-氧化物 $1(5 \mathrm{mmol}) 、$ 含不同取代基的噻唑化合物 2 (6 $\mathrm{mmol})$ 、无水氯化铁 $(0.97 \mathrm{~g}, 6 \mathrm{mmol})$ 、硅胶 $(2.44 \mathrm{~g})$ 和无 水 1,2 二氯乙烷 $(50 \mathrm{~mL})$. 氮气保护下, $100{ }^{\circ} \mathrm{C}$ 加热回流 $8 \mathrm{~h}$. 反应结束后, 冷却至室温, 减压蒸除溶剂, 粗产物 以硅胶柱层析分离 $[V($ 石油醚 $) / V(\mathrm{EtOAc})=10 / 1$ 洗脱 $]$ 得 到白色吡嗪一噻唑联芳化合物 $\mathbf{3 a} \sim \mathbf{3 h}$.

2-(3,6-二甲基吡嗪-2-基)噻唑(3a): 白色固体, 产率 $64 \%, R_{\mathrm{f}}=0.61$. m.p. $134 \sim 136{ }^{\circ} \mathrm{C} ;{ }^{1} \mathrm{H}$ NMR $(400 \mathrm{MHz}$, $\left.\mathrm{CDCl}_{3}\right) \delta: 8.34$ (s, $1 \mathrm{H}$, Pyrazine-H), $7.98(\mathrm{~d}, J=3.2 \mathrm{~Hz}$, $1 \mathrm{H}$, Thiazole-H), $7.46(\mathrm{~d}, J=3.2 \mathrm{~Hz}, 1 \mathrm{H}$, Thiazole-H), 3.00 (s, $\left.3 \mathrm{H}, \mathrm{CH}_{3}\right), 2.58$ (s, 3H, $\left.\mathrm{CH}_{3}\right) ;{ }^{13} \mathrm{C}$ NMR $(100 \mathrm{MHz}$, $\left.\mathrm{CDCl}_{3}\right) \delta: 169.2,150.1,148.2,144.1,143.7,143.2,121.8$, 23.4, 20.9; IR (KBr) v: 3100, 2918, 1496, 1435, 1339, 1320, 1177, 1028, 961, 874, $739 \mathrm{~cm}^{-1}$; HRMS-ESI calcd for $\mathrm{C}_{9} \mathrm{H}_{10} \mathrm{~N}_{3} \mathrm{~S}[\mathrm{M}+\mathrm{H}]^{+}$192.0590, found 192.0775.

2-(3,6-二甲基吡嗪-2-基)-4-甲基噻唑(3b): 白色固 体, 产率 59\%, $R_{\mathrm{f}}=0.60$. m.p. $167 \sim 169{ }^{\circ} \mathrm{C} ;{ }^{1} \mathrm{H}$ NMR $\left(400 \mathrm{MHz}, \mathrm{CDCl}_{3}\right) \delta: 8.32$ (s, 1H, Pyrazine-H), 7.01 (s, $1 \mathrm{H}$, Thiazole-H), 2.99 (s, 3H, $\left.\mathrm{CH}_{3}\right), 2.57\left(\mathrm{~s}, 3 \mathrm{H}, \mathrm{CH}_{3}\right)$, $2.53\left(\mathrm{~s}, 3 \mathrm{H}, \mathrm{CH}_{3}\right) ;{ }^{13} \mathrm{C} \mathrm{NMR}\left(100 \mathrm{MHz}, \mathrm{CDCl}_{3}\right) \delta: 167.9$, 154.3, 150.0, 148.1, 143.8, 143.0, 116.6, 23.4, 20.9, 17.5; IR (KBr) v: 3058, 2921, 1653, 1519, 1443, 1342, 1177, $1025,978,874,801 \mathrm{~cm}^{-1}$; HRMS-ESI calcd for $\mathrm{C}_{10} \mathrm{H}_{12-}$ $\mathrm{N}_{3} \mathrm{~S}[\mathrm{M}+\mathrm{H}]^{+}$206.0746, found 206.0755.

2-(3,6-二甲基吡嗪-2-基)-4,5-二甲基噻唑(3c): 白色 固体, 产率 54\%, $R_{\mathrm{f}}=0.60$. m.p. $181 \sim 183{ }^{\circ} \mathrm{C} ;{ }^{1} \mathrm{H}$ NMR $\left(400 \mathrm{MHz}, \mathrm{CDCl}_{3}\right) \delta: 8.27(\mathrm{~s}, 1 \mathrm{H}$, Pyrazine-H), $2.96(\mathrm{~s}$, $\left.3 \mathrm{H}, \mathrm{CH}_{3}\right), 2.54\left(\mathrm{~s}, 3 \mathrm{H}, \mathrm{CH}_{3}\right), 2.41\left(\mathrm{~s}, 3 \mathrm{H}, \mathrm{CH}_{3}\right), 2.40$ (s, $\left.3 \mathrm{H}, \mathrm{CH}_{3}\right) ;{ }^{13} \mathrm{C}$ NMR $\left(100 \mathrm{MHz}, \mathrm{CDCl}_{3}\right) \delta: 163.6,150.1$, 149.9, 147.9, 144.1, 142.4, 129.8, 23.4, 20.9, 15.0, 11.4; IR (KBr) $v: 2960,2921,1726,1653,1544,1446,1345,1280$, $1179,1025,952,899 \mathrm{~cm}^{-1}$; HRMS-ESI calcd for $\mathrm{C}_{11} \mathrm{H}_{14^{-}}$ $\mathrm{N}_{3} \mathrm{~S}\left[[\mathrm{M}+\mathrm{H}]^{+}\right.$220.0903, found 220.0908 . 2-(3,6-二甲基吡嗪-2-基)苯并噻唑(3d): 白色固体, 产率 76\%, $R_{\mathrm{f}}=0.61$. m.p. $181 \sim 183{ }^{\circ} \mathrm{C} ;{ }^{1} \mathrm{H}$ NMR $(400$ $\left.\mathrm{MHz}, \mathrm{CDCl}_{3}\right) \delta: 8.39$ (s, $1 \mathrm{H}$, Pyrazine-H), 8.11 (d, $J=8.0$, $\mathrm{Hz}, 1 \mathrm{H}, \mathrm{ArH}), 7.95$ (dd, $J=8.0,0.3 \mathrm{~Hz}, 1 \mathrm{H}, \mathrm{ArH}), 7.50$ $(\operatorname{td}, J=8.0,1.2 \mathrm{~Hz}, 1 \mathrm{H}, \mathrm{ArH}), 7.43(\mathrm{td}, J=8.0,1.2 \mathrm{~Hz}, 1 \mathrm{H}$, $\mathrm{ArH}), 3.12\left(\mathrm{~s}, 3 \mathrm{H}, \mathrm{CH}_{3}\right), 2.62$ (s, $\left.3 \mathrm{H}, \mathrm{CH}_{3}\right) ;{ }^{13} \mathrm{C} \mathrm{NMR}(100$ $\left.\mathrm{MHz}, \mathrm{CDCl}_{3}\right) \delta: 169.2,154.7,150.3,149.5,143.9,143.7$, 136.0, 126.1, 125.8, 124.0, 121.6, 23.6, 20.0; IR (KBr) $v$ : 2921, 2894, 1728, 1653, 1556, 1505, 1444, 1350, 1288, 1170, 1071, 1022, 1009, 958, 904, 765, 727, $687 \mathrm{~cm}^{-1}$; HRMS-ESI calcd for $\mathrm{C}_{13} \mathrm{H}_{12} \mathrm{~N}_{3} \mathrm{~S}[\mathrm{M}+\mathrm{H}]^{+} 242.0846$, found 242.0930

2-(5,6-二甲基吡嗪-2-基)噻唑(3e): 白色固体, 产率 $47 \%, R_{\mathrm{f}}=0.62$. m.p. $181 \sim 183{ }^{\circ} \mathrm{C} ;{ }^{1} \mathrm{H}$ NMR $(400 \mathrm{MHz}$, $\left.\mathrm{CDCl}_{3}\right) \delta: 9.11(\mathrm{~s}, 1 \mathrm{H}$, Pyrazine-H), $7.94(\mathrm{~d}, \mathrm{~J}=2.9 \mathrm{~Hz}$, 1H, Thiazole-H), $7.45(\mathrm{~d}, \mathrm{~J}=2.9 \mathrm{~Hz}, 1 \mathrm{H}$, Thiazole-H), $2.60\left(\mathrm{~s}, 3 \mathrm{H}, \mathrm{CH}_{3}\right), 2.60$ (s, $\left.3 \mathrm{H}, \mathrm{CH}_{3}\right) ;{ }^{13} \mathrm{C} \mathrm{NMR}(100 \mathrm{MHz}$, $\left.\mathrm{CDCl}_{3}\right) \delta: 167.2,153.3,152.0,144.2,143.7,137.4,121.3$, 22.0, 21.9; IR (KBr) v: 2920, 2850, 1653, 1558, 1454, 1384, 1149, 961, 905, 874, $745 \mathrm{~cm}^{-1}$; HRMS-ESI calcd for $\mathrm{C}_{9} \mathrm{H}_{10} \mathrm{~N}_{3} \mathrm{~S}[\mathrm{M}+\mathrm{H}]^{+}$192.0590, found 192.0719.

2-(5,6-二乙基吡嗪-2-基)噻唑(3f): 白色固体, 产率 $41 \%, R_{\mathrm{f}}=0.60$. m.p. $181 \sim 183{ }^{\circ} \mathrm{C} ;{ }^{1} \mathrm{H}$ NMR $(400 \mathrm{MHz}$, $\left.\mathrm{CDCl}_{3}\right) \delta: 9.14$ (s, 1H, Pyrazine-H), $7.94(\mathrm{~d}, \mathrm{~J}=3.2 \mathrm{~Hz}$, 1H, Thiazole-H), $7.44(\mathrm{~d}, \mathrm{~J}=3.2 \mathrm{~Hz}, 1 \mathrm{H}$, Thiazole-H), $2.91\left(\mathrm{q}, \mathrm{J}=7.5 \mathrm{~Hz}, 4 \mathrm{H}, \mathrm{CH}_{2}\right), 1.37(\mathrm{t}, \mathrm{J}=7.5 \mathrm{~Hz}, 3 \mathrm{H}$, $\left.\mathrm{CH}_{3}\right), 1.33$ (t, J=7.5 Hz, 3H, $\left.\mathrm{CH}_{3}\right) ;{ }^{13} \mathrm{C}$ NMR $(100 \mathrm{MHz}$, $\left.\mathrm{CDCl}_{3}\right) \delta: 167.7,157.2,155.5,144.2,143.5,137.3,121.2$, 27.5, 27.1, 12.7, 12.3; IR (KBr) v: 2918, 2948, 1729, 1656, 1460, 1446, 1308, 1280, 1177, 868, 722, $669 \mathrm{~cm}^{-1}$; HRMS-ESI calcd for $\mathrm{C}_{11} \mathrm{H}_{14} \mathrm{~N}_{3} \mathrm{~S}\left[[\mathrm{M}+\mathrm{H}]^{+} 220.0903\right.$, found 220.0912 .

2-(喹喔啉-2-基)噻唑(3g): 白色固体, 产率 $68 \%, R_{\mathrm{f}}$ $=0.59$. m.p. $181 \sim 183{ }^{\circ} \mathrm{C} ;{ }^{1} \mathrm{H}$ NMR $\left(400 \mathrm{MHz}, \mathrm{CDCl}_{3}\right) \delta$ : $9.75(\mathrm{~s}, 1 \mathrm{H}$, Pyrazine-H), 8.14 (td, J=5.0, $2.2 \mathrm{~Hz}, 2 \mathrm{H}$, ArH), $8.05(\mathrm{~d}, \mathrm{~J}=3.2 \mathrm{~Hz}, 1 \mathrm{H}$, Thiazole-H), $7.78 \sim 7.83(\mathrm{~m}$, 2H, ArH), $7.58\left(\mathrm{~d}, \mathrm{~J}=3.12 \mathrm{~Hz}, 1 \mathrm{H}\right.$, Thiazole-H); ${ }^{13} \mathrm{C}$ NMR $\left(100 \mathrm{MHz}, \mathrm{CDCl}_{3}\right) \delta: 167.0,145.8,144.7,142.8,142.5$, 141.7, 130.6, 130.4, 129.4, 129.4, 122.7; IR (KBr) v: 2921, 2850, 1650, 1493, 1457, 1415, 1364, 1306, 1129, 1014, $947,773,728 \mathrm{~cm}^{-1}$; HRMS-ESI calcd for $\mathrm{C}_{11} \mathrm{H}_{8} \mathrm{~N}_{3} \mathrm{~S}$ [[M+ $\mathrm{H}]^{+}$214.1433, found 214.1429.

4-甲基-2-(喹喔啉-2-基)噻唑(3h): 白色固体, 产率 $53 \%, R_{\mathrm{f}}=0.59$. m.p. $181 \sim 183{ }^{\circ} \mathrm{C} ;{ }^{1} \mathrm{H}$ NMR $(400 \mathrm{MHz}$, $\left.\mathrm{CDCl}_{3}\right) \delta: 9.71(\mathrm{~s}, 1 \mathrm{H}$, Pyrazine-H), $8.11 \sim 8.15(\mathrm{~m}, 2 \mathrm{H}$, 
ArH), $7.77 \sim 7.80(\mathrm{~m}, 2 \mathrm{H}, \mathrm{ArH}), 7.13(\mathrm{~s}, 1 \mathrm{H}$, Thiazole-H), 2.59 (s, $\left.3 \mathrm{H}, \mathrm{CH}_{3}\right) ;{ }^{13} \mathrm{C} \mathrm{NMR}\left(100 \mathrm{MHz}, \mathrm{CDCl}_{3}\right) \delta: 166.0$, $155.0,145.9,142.8,142.6,141.8,130.5,130.2,129.4$, 129.3, 117.6, 17.3; IR (KBr) v: 3083, 2918, 2850, 1648, $1547,1513,1491,1463,1437,1129,1020,972,930,759$ $\mathrm{cm}^{-1}$; HRMS-ESI calcd for $\mathrm{C}_{12} \mathrm{H}_{10} \mathrm{~N}_{3} \mathrm{~S}\left[[\mathrm{M}+\mathrm{H}]^{+}\right.$ 228.1590 , found 228.1596 .

\section{3 抗氧化活性测试}

\subsection{1 抑制 AAPH 引发 DNA 氧化反应测试}

按照文献已报道的方法 ${ }^{[17]}$. 在 $50 \mathrm{~mL}$ 雉形瓶中, 加 入 $26.8 \mathrm{~mL} 2.24 \mathrm{~g} / \mathrm{L}$ DNA 溶液、 $3 \mathrm{~mL} 400 \mathrm{mmol} / \mathrm{L} \mathrm{AAPH}$ 溶液和 $0.2 \mathrm{~mL}$ 待测化合物储备液 $(0 、 15 、 30 、 45$ 或 60 $\mathrm{mmol} / \mathrm{L})$, 充分混匀后置于 $37{ }^{\circ} \mathrm{C}$ 水浴中, 不同时刻 $(0$ 、 $1 、 2 、 4 、 6 、 8 、 10$ 和 $12 \mathrm{~h}$ )从中移取 $2 \mathrm{~mL}$, 再加入 $1 \mathrm{~mL}$ $1.0 \% \mathrm{TBA}$ 溶液和 $1 \mathrm{~mL} 3.0 \% \mathrm{TCA}$ 溶液, 于 $100{ }^{\circ} \mathrm{C}$ 水浴 加热 $15 \mathrm{~min}$, 冷却至室温, 加入 $1.5 \mathrm{~mL}$ 正丁醇充分振荡 萃取 TBARS, 在 $535 \mathrm{~nm}$ 下测定正丁醇的吸光度 A.

以吸光度为纵坐标, 反应时间为横坐标作图, 如图 1 所示. 按照文献方法 ${ }^{[18]}$ 求得抗氧化活性化合物的抑制 期 $\left(t_{\mathrm{inh}}\right)$, 以抑制期 $\left(t_{\mathrm{inh}}\right)$ 为纵坐标, 化合物浓度为横坐标 作图, 如图 2 所示. 文献报道 ${ }^{[19]}$ 抗氧化剂产生的抑制期 $\left(t_{\mathrm{inh}}\right)$ 与浓度呈线性关系, 即如方程式(1)所示:

$$
t_{\text {inh }}=\left(n / R_{\mathrm{i}}\right) c_{\text {antioxidant }}
$$

$n$ 为抗氧化剂的有效计量因子, 表示单个抗氧化分子淬 灭自由基的数量; $R_{\mathrm{i}}$ 为从 $\mathrm{AAPH}$ 分解产生的过氧自由基 引发底物氧化反应的速率, 等于 $3.36 \mu \mathrm{mol} /(\mathrm{L} \cdot \mathrm{min})^{[20]}$.

由化合物抑制期 $\left(t_{\mathrm{inh}}\right)$ 和浓度，通过线性拟合可得到 抑制期 $\left(t_{\text {inh }}\right)$ 与浓度之间的方程式, 见表 2 , 结合方程式 (1)可求得化合物的有效计量因子，见表 2 .

\subsection{2 抑制 $\mathrm{HO} ・$ 和 $\mathrm{GS}$ •引发 DNA 氧化反应测试}

按照文献方法 ${ }^{[21]}$ 对化合物抑制 HO•引发 DNA 氧化 反应性能进行测试. 在 $50 \mathrm{~mL}$ 锥形瓶中, 加入 $13.4 \mathrm{~mL}$ $2.24 \mathrm{~g} / \mathrm{L}$ DNA 溶液、 $1.0 \mathrm{~mL} 30 \mathrm{mmol} / \mathrm{L}$ 的 $\mathrm{H}_{2} \mathrm{O}_{2}$ 溶液、 $0.5 \mathrm{~mL} 120 \mathrm{mmol} / \mathrm{L} \mathrm{TCHQ}$ 溶液和 $0.1 \mathrm{~mL} 30 \mathrm{mmol} / \mathrm{L}$ 待 测化合物储备液, 充分混匀于 $37{ }^{\circ} \mathrm{C}$ 水浴 $30 \mathrm{~min}$. 从中 移取 $2 \mathrm{~mL}$, 再加入 $1 \mathrm{~mL} 1 \% \mathrm{TBA}$ 溶液和 $1 \mathrm{~mL} 3 \% \mathrm{TCA}$ 溶液, 于 $100{ }^{\circ} \mathrm{C}$ 水浴加热 $30 \mathrm{~min}$, 冷却至室温, 加入 1.5 $\mathrm{mL}$ 正丁醇萃取 TBARS, 在 $535 \mathrm{~nm}$ 下测定正丁醇的吸 光度. 空白实验和加入化合物后的 TBARS 吸光度值分 别为 $A_{0}$ 和 $A$, 化合物的抗氧化能力以 $A / A_{0} \times 100 \%$ 来表 示, 即化合物相对空白 TBARS 吸光度百分数.

按照文献方法 ${ }^{[22]}$ 对化合物抑制 GS・引发 DNA 氧化 反应性能进行测试. 在 $50 \mathrm{~mL}$ 锥形瓶中, 加入 $13.4 \mathrm{~mL}$ $2.24 \mathrm{~g} / \mathrm{L}$ DNA 溶液、 $1.0 \mathrm{~mL} 75 \mathrm{mmol} / \mathrm{L}$ 的 $\mathrm{CuSO}_{4}$ 溶液、
$0.5 \mathrm{~mL} 90 \mathrm{mmol} / \mathrm{L} \mathrm{GSH}$ 溶液和 $0.1 \mathrm{~mL} 30 \mathrm{mmol} / \mathrm{L}$ 待测 化合物储备液, 充分混匀于 $37{ }^{\circ} \mathrm{C}$ 水浴 $90 \mathrm{~min}$. 从中移 取 $2 \mathrm{~mL}$, 再加入 $1.0 \mathrm{~mL} 30 \mathrm{mmol} / \mathrm{L}$ EDTA 溶液、 $1 \mathrm{~mL}$ $1.0 \% \mathrm{TBA}$ 溶液和 $1 \mathrm{~mL} 3 \% \mathrm{TCA}$ 溶液, 于 $100{ }^{\circ} \mathrm{C}$ 水浴加 热 $30 \mathrm{~min}$, 冷却至室温, 加入 $1.5 \mathrm{~mL}$ 正丁醇萃取 TBARS，在 $535 \mathrm{~nm}$ 下测定正丁醇的吸光度. 采用抑制 $\mathrm{HO}$ •引发 DNA 氧化反应分析方法，可得化合物抑制 GS・ 引发 DNA 氧化反应的相对空白 TBARS 吸光度百分数.

\section{4 还原自由基性能测试}

按照文献方法 ${ }^{[23]}$ 对化合物淬灭 ABTS・性能进行测 试. 移取 $1.9 \mathrm{~mL} \mathrm{ABTS}$ •乙醇溶液 (吸光度为 0.961 ), 加入 $0.1 \mathrm{~mL} 1 \mathrm{mmol} / \mathrm{L}$ 待测化合物储备液, 待测化合物终浓 度为 $50 \mu \mathrm{mol} / \mathrm{L}$, 混合均匀, 在 $734 \mathrm{~nm}$ 测定 $0 \sim 1800 \mathrm{~s}$ 吸光度. 由朗伯比尔定理可求得各时刻 ABTS 的浓度, 以 ABTS•浓度为纵坐标，反应时间为横坐标，作 ABTS• 浓度随时间的衰减曲线.

按照文献方法 ${ }^{[24]}$ 对化合物淬灭 DPPH・性能进行测 试. 移取 $1.9 \mathrm{~mL} \mathrm{DPPH}$ •乙醇溶液 (吸光度为 1.076 ), 加入 $0.1 \mathrm{~mL} 2 \mathrm{mmol} / \mathrm{L}$ 待测化合物储备液, 待测化合物终浓 度为 $100 \mu \mathrm{mol} / \mathrm{L}$, 混合均匀, 在 $517 \mathrm{~nm}$ 测定 $0 \sim 1800 \mathrm{~s}$ 吸光度. 采用与淬灭 ABTS・分析方法, 作 DPPH・浓度随 时间的衰减曲线。

\section{5 数据统计与分析}

采用 Origin 8.0 软件对所得数据进行 ANOVA 方差 分析，显著性检验方法为 Duncan 多重检验，显著水平 为 0.05 .

辅助材料(Supporting Information) 吡嗪一噻唑联芳化 合物 3a 3h 的 ${ }^{1} \mathrm{H} N M R 、{ }^{13} \mathrm{C} N M R$ 和 MS 谱图, 这些材 料可以免费从本刊网站(http://sioc-journal.cn/)上下载.

\section{References}

[1] (a) Abu-Hashem, A. A.; El-Shazly, M. Med. Chem. 2018, 14, 356. (b) Zaki, R. M.; Kamal El-Dean, A. M.; Radwan, S. M.; Abdul-Malik, M. A. Curr. Org. Synth. 2018, 6, 863.

(c) Shankar, B.; Jalapathi, P.; Valeru, A.; Kumar, A. K.; Saikrishna, B.; Kudle, K. R. Med. Chem. Res. 2017, 26, 1835.

[2] (a) Srinivasa, S. B.; Poojary, B.; Brahmavara, U.; Das, A. J.; Middha, S. K. ChemistrySelect 2018, 44. 12478.

(b) Sinha, S.; Doble, M.; Manju, S. L. Eur. J. Med. Chem. 2018 , 158,34

(c) Rajendran, N. D.; Stephen, A. D.; Jelsch, C.; Escudero-Adan, E. C. Croat. Chem. Acta 2018, 2, 221.

(d) Leleu-Chavain, N.; Baudelet, D.; Heloire, V. M.; Rocha, D. E.; Renault, N.; Barczyk, A.; Djouina, M.; Body-Malapel, M.; Carato, P.; Millet, R. Eur. J. Med. Chem. 2019, 165, 347.

[3] (a) Kusstatscher, P.; Cernava, T.; Liebminger, S.; Berg, G. Sci. Rep. 2017, 7, 13253.

(b) Zitko, J.; Dolezal, M.; Svobodova, M.; Vejsova, M.; Kunes, J.; Kucera, R.; Jilek, P. Bioorg. Med. Chem. 2011, 19, 1471.

[4] (a) Reddy, N. B.; Zyryanov, G. V.; Reddy, G. M.; Balakrishna, A.; 
Padmaja, A.; Padmavathi, V.; Reddy, C. S.; Garcia, J. R.; Sravya, G. J. Heterocycl. Chem. 2019, $2,589$.

(b) Masood, M. M.; Irfan, M.; Alam, S.; Hasan, P.; Queen, A.; Shahid, S.; Zahid, M.; Azam, A.; Abid, M. Russ. Lett. Drug. Des. Discovery 2019, 2, 160.

(c) Panchani, N. M.; Joshi, H. S. Russ. Lett. Drug. Des. Discovery 2019, 3, 284.

(d) Edrees, M. M.; Abu-Melha, S.; Saad, A. M.; Kheder, N. A.; Gomha, S. M.; Muhammad, Z. A. Molecules 2018, 11, 2970.

[5] Gabr, I. M.; El-Asmy, H. A.; Emmam, M. S.; Mostafa, S. I. Transition Metal. Chem. 2009, 34, 409.

[6] (a) Sharma, A.; Gudala, S.; Ambati, S. R.; Mahapatra, S. P.; Raza, A.; Aruna, L. V.; Payra, S.; Jha, A.; Kumar, A.; Penta, S. ChemistrySelect 2018, 39, 11012.

(b) Bhatt, P.; Kumar, M.; Jha, A. Mol. Diversity 2018, 4, 827.

(c) Biswas, N. M.; Shard, A.; Patel, S.; Sengupta, P. Drug Dev. Res. 2018, 8, 391 .

(d) Zhang, Z. H.; Wu, H. M.; Deng, S. N.; Chai, R. X.; Mwenda, M. C.; Peng, Y. Y.; Cai, D.; Chen, Y. Chem. Pap. 2019, 2, 355.

[7] Kucerova-Chlupacova, M.; Dosedel, M.; Kunes, J.; SoltesovaPrnova, M.; Majekova, M.; Stefek, M. Monatsh. Chem. 2018, 149, 921.

[8] (a) Uremis, N.; Uremis, M. M.; Tolun, F. I.; Ceylan, M.; Doganer, A.; Kurt, A. H. J. Anticancer. Res. 2017, 11, 6381.

(b) Parasotas, I.; Anusevicius, K.; Vaickelioniene, R.; Jonuskiene, I.; Stasevych, M.; Zvarych, V.; Komarovska-Porokhnyavets, O.; Novikov, V.; Belyakov, S.; Mickevicius, V. ARKIVOC 2018, 3, 240. (c) Djukic, M.; Fesatidou, M.; Xenikakis, I.; Geronikaki, A.; Angelova, V. T.; Savic, V.; Pasic, M.; Krilovic, B.; Djukic, D.; Gobeljic, B. Chem.-Biol. Interact. 2018, 286, 119.

[9] (a) Sebastian, S. H. R.; Al-Alshaikh, M. A.; El-Emam, A. A.; Panicker, C. Y.; Zitko, J.; Dolezal, M.; VanAlsenoy, C. J. Mol. Struct.
2016, $1119,188$.

(b) Wu, H. M.; Zhou, K.; Wu, T.; Cao, Y. G. Chem. Biol. Drug Des. 2016, 88,411

[10] (a) Wang, Y. Y.; Wu, C. L.; Zhang, Q. J.; Shan, Y.; Gu, W.; Wang, S. F. Bioorg. Chem. 2019, 84, 468.

(b) George, R. F.; Samir, E. M.; Abdelhamed, M. N.; Abdel-Aziz, H. A.; Abbas, S. E. S. Bioorg. Chem. 2019, 83, 186.

(c) Prajapati, N. P.; Patel, K. D.; Vekariya, R. H.; Patel, H. D.; Rajani, D. P. J. Mol. Struct. 2019, 1179, 401.

[11] (a) Khake, S. M.; Soni, V.; Gonnade, R. G.; Punji, B.; Dalton Trans. 2014, 43,16084.

(b) Dowlut, M.; Mallik, D.; Organ, M. G. Chem.-Eur. J. 2010, 16, 4279.

[12] (a) Pandey, D. K.; Khake, S. M.; Gonnade, R. G.; Punji, B. RSC Adv. 2015, 5, 81502 .

(b) Khake, S. M.; Jagtap, R. A.; Dangat, Y. B.; Gonnade, R. G.; Vanka, K.; Punji, B. Organometallics 2016, 35, 875.

[13] Wu, Z. Y.; Chang, C. C.; Tang, X. T.; Liu, S.; Xi, G. L.; Zhao, M. Q.; Liu, P. F. ChemistrySelect 2018, 3, 13038.

[14] Zhang, X. P.; Chen, Z. F.; Han, L. Fine Chem. 2020, 37: 1461.

[15] Xiao, C.; Song, Z. G.; Liu, Z. Q. Eur. J. Med. Chem. 2010, 45: 2559.

[16] Stojanoić, S.; Brede, O. Phys. Chem. Chem. Phys. 2002, 4, 757.

[17] Xi, G. L.; Liu, Z. Q. J. Agric. Food Chem. 2015, 63, 3516.

[18] (a) Xi, G. L.; Liu, Z. Q. Tetrahedron 2014, 70, 8397. (b) Xi, G. L.; Liu, Z. Q. Tetrahedron 2015, 71, 9602.

[19] He, J. H.; Li, J. Z.; Liu, Z. Q. Med. Chem. Res. 2013, 22, 2847.

[20] Nabi, G.; Liu, Z. Q. Med. Chem. Res. 2012, 21, 3015.

[21] Zhao, C.; Liu, Z. Q. Biochimie 2013, 95, 842.

[22] Wang, R.; Liu, Z. Q. Med. Chem. Res. 2013, 22, 1563.

[23] Li, G. X.; Liu, Z. Q.; Luo, X. Y. Eur. J. Med. Chem. 2010, 45, 1821.

[24] Xi, G. L.; Liu, Z. Q. Eur. J. Med. Chem. 2013, 68, 385.

(Li, L.; Fan, Y.) 